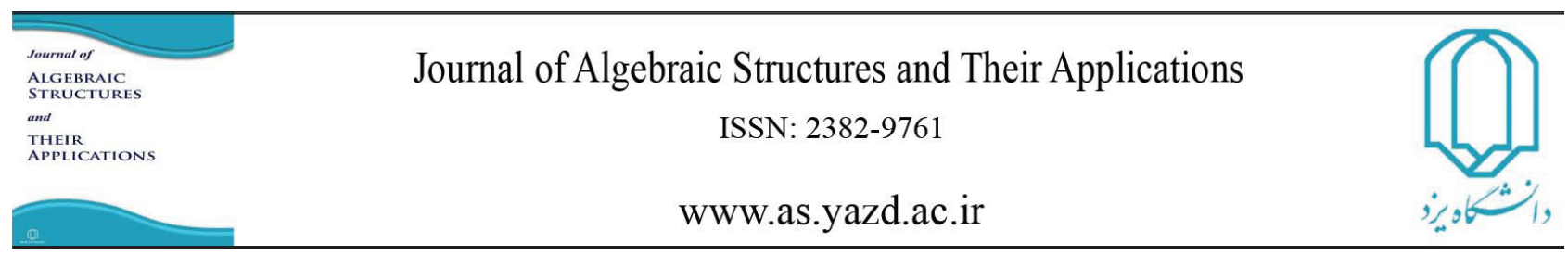

Algebraic Structures and Their Applications Vol. 6 No. 1 ( 2019 ) pp 115-125.

\title{
A NOTE ON DERIVATIONS IN RINGS AND BANACH ALGEBRAS
}

\author{
NADEEM UR REHMAN, SHULIANG HUANG* AND MOHD ARIF RAZA
}

\begin{abstract}
Let $R$ be a prime ring with $U$ the Utumi quotient ring and $Q$ be the Martindale quotient ring of $R$, respectively. Let $d$ be a derivation of $R$ and $m, n$ be fixed positive integers. In this paper, we study the case when one of the following holds: $(i) d(x) \circ_{n} d(y)=x \circ_{m} y$ (ii) $d(x) \circ_{m} d(y)=(d(x \circ y))^{n}$ for all $x, y$ in some appropriate subset of $R$. We also examine the case where $R$ is a semiprime ring. Finally, as an application we apply our result to the continuous derivations on Banach algebras.
\end{abstract}

\section{INTRODUCTION AND PRELIMINARIES}

In all that follows, unless specifically stated otherwise, $R$ will be an associative ring, $Z(R)$ the center of $R, Q$ its Martindale quotient ring and $U$ its Utumi quotient ring. The center of $U$, denoted by $C$, is called the extended centroid of $R$ (we refer the reader to [3, Chapter 2], for the definitions and related properties of these objects). For any $x, y \in R$, the symbol $[x, y]$ and $x \circ y$ stands for the commutator $x y-y x$ and anti-commutator $x y+y x$, respectively. Given

DOI : $10.29252 /$ as. 2019.1378

MSC(2010): Primary: 46J10, 16N20, 16N60, 16W25.

Keywords: Prime and semiprime rings, Derivations, Martindale ring of quotients, Banach algebras, Radical.

Received: 28 Feb 2019, Accepted: 04 May 2019.

*Corresponding author

The second author was supported by the Anhui Provincial Natural Science Foundation (No. 1808085MA14) and the Key University Science Research Project of Anhui Province (No. KJ2018A0433) and also Research Project of Chuzhou University (No. zrjz2017005) of P. R. China.

(C) 2019 Yazd University. 
$x, y \in R$, we set $x \circ_{0} y=x, x \circ_{1} y=x \circ y=x y+y x$, and inductively $x \circ_{m} y=\left(x \circ_{m-1} y\right) \circ y$ for $m>1$. Recall that a ring $R$ is prime if $x R y=\{0\}$ implies either $x=0$ or $y=0$, and $R$ is semiprime if $x R x=\{0\}$ implies $x=0$. An additive mapping $d: R \rightarrow R$ is called a derivation if $d(x y)=d(x) y+x d(y)$ holds for all $x, y \in R$. In particular $d$ is an inner derivation induced by an element $q \in R$, if $d(x)=[q, x]$ holds for all $x \in R$.

During the past few decades, there has been an ongoing interest concerning the relationship between the commutativity of a ring and the existence of certain specific types of derivations (see[2], where further references can be found). In [2, Theorem 4.1], Ashraf and Rehman proved that if $R$ is a prime ring, $I$ a nonzero ideal of $R$ and $d$ is a nonzero derivation of $R$ such

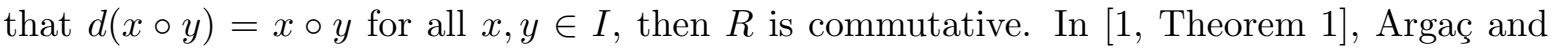
Inceboz generalized the above result and they proved the following: Let $R$ be a prime ring, $I$ a nonzero ideal of $R$, and $n$ a fixed positive integer. If $R$ admits a nonzero derivation $d$ with the property $d(x \circ y)^{n}=x \circ y$ for all $x, y \in I$, then $R$ is commutative.

In 1994 Bell and Daif [4, Theorem 1] initiated the study of strong commutativity-preserving (scp) maps and proved that a nonzero right ideal $I$ of a semiprime ring is central if $R$ admits a derivation which is scp on $I$. In 2002 Ashraf and Rehman [2, Theorem 4.4] also proved that if $R$ is a 2-torsion free prime ring, $I$ a nonzero ideal of $R$ and $d$ is a nonzero derivation of $R$ such that $d(x) \circ d(y)=x \circ y$ for all $x, y \in I$, then $R$ is commutative. The present paper is motivated by the previous results and we here shall generalize the results obtained in [I] and [2]. Moreover, we continue this line of investigation by examining what happens for a ring $R$ (or an algebra $\mathfrak{A}$ ) satisfying one of the following identities:

(i) $d(x) \circ_{n} d(y)=x \circ_{m} y$;

(ii) $d(x) \circ_{m} d(y)=(d(x \circ y))^{n}$ for all $x, y \in I$.

We obtain some analogous results for semiprime rings in the case $I=R$. Finally, by using the above identities we obtain some range inclusion results on non-commutative Banach algebras.

In the last section of this paper we will consider $\mathfrak{A}$ as a Banach algebra with Jacobson radical $\operatorname{rad}(\mathfrak{A})$. The classical result of Singer and Wermer in [17, Theorem 1] said that any continuous derivation on a commutative Banach algebra has the range in the Jacobson radical of the algebra. Singer and Wermer also formulated the conjecture that the continuity assumption can be removed. In 1988 Thomas [18] verified this conjecture. It is clear that the same result of Singer-Wermer does not hold in non-commutative Banach algebras (because of inner derivations).

Hence in this context a very interesting question is how to obtain the non-commutative version of the Singer-Wermer theorem. A first answer to this problem was obtained by Sinclair in [16, Theorem 2.2]. He proved that every continuous derivation of a Banach algebra leaves 
primitive ideals of the algebra invariant. Since then many authors obtained more information about derivations satisfying certain suitable conditions in Banach algebras. Here we will continue the investigation about the relationship between the structure of an algebra $\mathfrak{A}$ and the behaviour of derivations defined on $\mathfrak{A}$. Then we apply our results on prime rings to the study of analogous conditions for continuous derivations on non-commutative Banach algebras.

\section{The Results in Prime Rings}

We will make frequent use of the following result due to Kharchenko [1], Theorem 2]:

Let $R$ be a prime ring, $d$ a nonzero derivation of $R$ and $I$ a nonzero two sided ideal of $R$. Let $f\left(x_{1}, \cdots x_{n}, d\left(x_{1}\right), \cdots, d\left(x_{n}\right)\right)$ be a differential identity on $I$, that is

$$
f\left(r_{1}, \cdots r_{n}, d\left(r_{1}\right), \cdots, d\left(r_{n}\right)\right)=0 \text { for all } r_{1}, \cdots, r_{n} \in I
$$

Then one of the following holds:

(1) either $d$ is an inner derivation on $Q$, the Martindale quotient ring of $R$, in the sense that there exists $q \in Q$ such that $d(x)=a d(q)(x)=[q, x]$ for all $x \in R$, and $I$ satisfies the generalized polynomial identity

$$
f\left(r_{1}, \cdots, r_{n},\left[q, r_{1}\right], \cdots,\left[q, r_{n}\right]\right)=0 \text { for all } r_{1}, \cdots, r_{n} \in I
$$

(2) or, $I$ satisfies the generalized polynomial identity

$$
f\left(x_{1}, \cdots, x_{n}, y_{1}, \cdots, y_{n}\right)=0 \text { for all } x_{1}, \cdots, x_{n}, y_{1}, \cdots, y_{n} \in I \text {. }
$$

Remark 2.1. Let $R$ be a prime ring and $I$ a nonzero ideal of $R$. If $I$ satisfies the polynomial $(x y+y x)^{n}=0$ for all $x, y \in I$. Then $R$ is commutative.

Proof. By the given hypothesis $(x y+y x)^{n}=0$ for all $x, y \in I$. It is obvious that, if $\operatorname{char}(R) \neq 2$, then $\left(2 x^{2}\right)^{n}=0$ for all $x \in I$. This is a contradiction by $\mathrm{Xu}$ [1.9, Lemma 3.2.1]. And if $\operatorname{char}(R)=2$, then $(x y+y x)^{n}=0=[x, y]^{n}$ for all $x, y \in I$. By Herstein [8, Theorem 2], we have $I \subseteq Z(R)$, and so $R$ is commutative by Mayne [15, Theorem 1].

Theorem 2.2. Let $R$ be a prime ring with characteristic different from 2 , I a nonzero ideal of $R$ and $m, n$ be fixed positive integers. If $R$ admits a nonzero derivationd such that $d(x) \circ_{n} d(y)=$ $x \circ_{m} y$ for all $x, y \in I$, then $R$ is commutative.

Proof. By our hypothesis, we have $d(x) \circ_{n} d(y)=x \circ_{m} y$ for all $x, y \in I$. This condition is a differential identity satisfied by $I$. By using Kharchenko's theorem [11, Theorem 2], either 
$d=a d(q)$ is the inner derivation induced by an element $q \in Q$, where $Q$ is the Martindale quotient ring of $R$, or $I$ satisfy the polynomial identity

$$
s \circ_{n} t=x \circ_{m} y \text { for all } x, y, s, t \in I \text {. }
$$

In the latter case set $y=0$ to obtain the identity $s \circ_{n} t=0$ for all $s, t \in I$. Then we get a contradiction as we have just seen. Assume now that $d=a d(q)$. Then $\left([q, x] \circ_{n}[q, y]\right)=x \circ_{m} y$ for any $x, y \in I$. Since by [5, Theorems 2], $I$ and $Q$ satisfy the same generalized polynomial identities, we have $\left([q, x] \circ_{n}[q, y]\right)=x \circ_{m} y$ for any $x, y \in Q$. Moreover, since $Q$ remains prime by the primeness of $R$, replacing $R$ by $Q$ we may assume that $q \in R$ and $C$ is just the center of $R$. Note that $R$ is centrally closed prime $C$-algebra in the present situation [7, Theorems 2.5 and 3.5], that is, $R C=R$. By Martindale's theorem in [13, Theorem 3], $R C$ (and so $R$ ) is a primitive ring. Since $R$ is primitive, there exists a vector space $\mathcal{V}$ and division $\operatorname{ring} \mathcal{D}$ such that $R$ is a dense ring of $D$-linear transformations over $\mathcal{V}$. Assume that $\operatorname{dim}_{\mathcal{D}} \mathcal{V} \geq 2$, otherwise we are done.

Our aim is to show that for any $v \in \mathcal{V}, v$ and $q v$ are linearly $\mathcal{D}$-dependent. If $v=0$, then $\{v, q v\}$ is $\mathcal{D}$-dependent. So we may assume that $v \neq 0$. So $v$ and $q v$ are linearly $\mathcal{D}$-independent for some $q \in \mathcal{D}$. Now we consider the two cases:

If $q^{2} v \notin \operatorname{Span}_{\mathcal{D}}\{v, q v\}$ then $\left\{v, q v, q^{2} v\right\}$ are linearly $\mathcal{D}$-independent. By the density of $R$ there exist $x, y \in R$ such that

$$
\begin{array}{ll}
x v=-v, & x q v=q v, \\
y v=0, & x q^{2} v=0, \\
y v=v, & y q^{2} v=v .
\end{array}
$$

We can easily see that $0=\left([q, x] \circ_{n}[q, y]\right) v-\left(x \circ_{m} y\right) v=(-1)^{n+1} 2^{n} v \neq 0$, a contradiction.

If $q^{2} v \in \operatorname{Span}_{\mathcal{D}}\{v, q v\}$ then $q^{2} v=v \alpha+q v \gamma$ for some $\alpha, 0 \neq \gamma \in \mathcal{D}$. In view of the density of $R$, there exist $x, y \in R$ such that

$$
\begin{aligned}
& x v=-v, \quad x q v=q v, \\
& y v=0, \quad y q v=v .
\end{aligned}
$$

Hence we get $0=\left([q, x] \circ_{n}[q, y]\right) v-\left(x \circ_{m} y\right) v=(-1)^{n+1} 2^{n} v \gamma^{n} \neq 0$, a contradiction. So $v$ and $q v$ are linearly $\mathcal{D}$-dependent for all $v \in \mathcal{V}$.

Now we want to show that $q v=v \mu(v)$, where $\mu(v) \in \mathcal{D}$ depends upon $v \in \mathcal{V}$. We claim that $\mu(v)$ is independent of the choice of $v \in V$ (see the proof of [ [ , Theorem 1]). If $v$ and $w$ are $\mathcal{D}$-dependent, say $v=w \beta$, where $\beta \in \mathcal{D}$, then $v \mu(v)=q v=q w \beta=w \mu(w) \beta=v \mu(w)$ and so $\mu(v)=\mu(w)$ as claimed. So, there exists $\lambda \in \mathcal{D}$ such that $q v=v \lambda$ for all $v \in V$. Now for $r \in R, v \in V$, we have $(r q) v=r(q v)=r(v \lambda)=(r v) \lambda=q(r v)=(q r) v$, that is $[q, R] V=0$. Since $V$ is a left faithful irreducible $R$-module, hence $[q, R]=0$, i.e. $q \in Z(R)$ and $d=0$, a contradiction. This finishes the proof of the theorem. 
Alg. Struc. Appl. Vol. 6 No. 1 (2019) 115-125.

Theorem 2.3. Let $R$ be a prime ring with characteristic different from $2, I$ a nonzero ideal of $R$ and $m, n$ be fixed positive integers. If $R$ admits a nonzero derivation d such that $d(x) \circ_{m}$ $d(y)=(d(x \circ y))^{n}$ for all $x, y \in I$, then $R$ is commutative.

Proof. Since $R$ is a prime ring with characteristic different from 2, we have given that

$$
d(x) \circ_{m} d(y)=(d(x \circ y))^{n} \text { for all } x, y \in I,
$$

which can be rewritten as

$$
d(x) \circ_{m} d(y)=(d(x) y+x d(y)+d(y) x+y d(x))^{n} \text { for all } x, y \in I .
$$

In the light of Kharcchenko's theorem [1], Theorem 2], we divide the proof into two cases:

Case 1. If the derivation $d$ is not inner, then $I$ satisfies the polynomial identity

$$
\left(s \circ_{m} t\right)=(s y+x t+t x+y s)^{n} \text { for all } x, y, s, t \in I .
$$

In particular, for $s=0, I$ satisfies the blended component $(t x+x t)^{n}=0$, for all $x, t \in I$. Then $R$ is commutative, by using the argument presented in Remark 2.1.

Case 2. Let now $d$ be an inner derivation induced by an element $q \in Q$, that is $d(x)=[q, x]$ for all $x \in R$. Then for any $x, y \in I$,

$$
[q, x] \circ_{m}[q, y]=([q, x] y+y[q, x]+[q, y] x+x[q, y])^{n}
$$

By Chuang [5, Theorem 2], $I$ and $Q$ satisfy same generalized polynomial identities (GPIs), we have

$$
[q, x] \circ_{m}[q, y]=([q,(x \circ y)])^{n} \text { for all } x, y \in Q
$$

In case the center $C$ of $Q$ is infinite, we have

$$
[q, x] \circ_{m}[q, y]=([q,(x \circ y)])^{n}
$$

for all $x, y \in Q \otimes_{C} \bar{C}$, where $\bar{C}$ is algebraic closure of $C$. Since both $Q$ and $Q \otimes_{C} \bar{C}$ are prime and centrally closed [ [7, Theorems 2.5 and 3.5], we may replace $R$ by $Q$ or $Q \otimes_{C} \bar{C}$ according as $C$ is finite or infinite. Thus we may assume that $R$ is centrally closed over $C$ (i.e., $R C=R$ ) which is either finite or algebraically closed and

$$
[q, x] \circ_{m}[q, y]=([q,(x \circ y)])^{n} \text { for all } x, y \in R
$$

By Martindale [ [1.3, Theorem 3], $R C$ (and so $R$ ) is a primitive ring with $\mathcal{D}$ as the associated division ring. Hence by Jacobson's theorem [9, p.75], $R$ is isomorphic to a dense ring of linear transformations of some vector space $\mathcal{V}$ over $\mathcal{D}$. If $\mathcal{V}$ is finite dimensional over $\mathcal{D}$, then the 
density of $R$ on $\mathcal{V}$ implies that $R \cong \mathcal{M}_{k}(\mathcal{D})$, where $M_{k}(\mathcal{D})$ is the ring of $k \times k$ matrices over $D$ and $k=d i m_{\mathcal{D}} \mathcal{V}$. Suppose that $\operatorname{dim}_{\mathcal{D}} \mathcal{V} \geq 2$, otherwise we are done.

We want to show that, for any $v \in \mathcal{V}, v$ and $q v$ are linearly $\mathcal{D}$-dependent. Since $q v=0$ implies that $\{v, q v\}$ is linearly $\mathcal{D}$-dependent, we can suppose that $q v \neq 0$. Suppose on contrary that $v$ and $q v$ are linearly $\mathcal{D}$-independent for some $q \in \mathcal{D}$. Similar to the proof of Theorem 2.2 , one can give a contradiction.

The following example demonstrates that $R$ to be prime is essential in the hypothesis.

Example 2.4. Let $S$ be any ring.

(i) Let $R=\left\{\left(\begin{array}{ll}a & b \\ 0 & 0\end{array}\right): a, b \in S\right\}$ and $I=\left\{\left(\begin{array}{ll}0 & a \\ 0 & 0\end{array}\right): a \in S\right\}$. Denote $e_{i j}$ the usual matrix unit with 1 in $(i, j)$-entry and zero elsewhere. We define a map $d: R \rightarrow R$ by $d(x)=e_{11} x-x e_{11}$. Then it is easy to see that $d$ is a nonzero derivation and $I$ is a nonzero ideal of $R$. For all positive integers $m, n$, it is obvious that $d$ satisfies the properties $d(x) \circ_{n} d(y)=\left(\begin{array}{ll}0 & 0 \\ 0 & 0\end{array}\right)=x \circ_{m} y$ and $d(x) \circ_{m} d(y)=\left(\begin{array}{ll}0 & 0 \\ 0 & 0\end{array}\right)=(d(x \circ y))^{n}$ for $x, y \in I$. However, $R$ is not commutative.

(ii) Let $R=\left\{\left(\begin{array}{ll}a & b \\ 0 & c\end{array}\right): a, b, c \in S\right\}$ and $I=\left\{\left(\begin{array}{ll}0 & a \\ 0 & 0\end{array}\right): a \in S\right\}$. Define a map $d: R \rightarrow R$ by $d(x)=\left[x, e_{11}+e_{12}\right]$. Then $R$ is a ring under usual operations. It is easy to see that $d$ is a nonzero derivation and $I$ is a nonzero ideal of $R$. It is straightforward to check that for all positive integers $m, n, d$ satisfies the properties $d(x) \circ_{n} d(y)=\left(\begin{array}{ll}0 & 0 \\ 0 & 0\end{array}\right)=x \circ_{m} y$ and $d(x) \circ_{m} d(y)=\left(\begin{array}{ll}0 & 0 \\ 0 & 0\end{array}\right)=(d(x \circ y))^{n}$ for $x, y \in I$, but $R$ is not commutative.

\section{The Results in Semiprime Rings}

In all that follows, $R$ will be a semiprime ring, $U$ the left Utumi quotient ring of $R$. In order to prove the main result of this section we will make use of the following lemmas:

Lemma 3.1. [3, Proposition 2.5.1] Any derivation of a semiprime ring $R$ can be uniquely extended to a derivation of its left Utumi quotient ring $U$, and so any derivation of $R$ can be defined on the whole $U$.

Lemma 3.2. [6, p. 38] If $R$ is semiprime then so is its left Utumi quotient ring. The extended centroid $C$ of a semiprime ring coincides with the center of its left Utumi quotient ring. 
Alg. Struc. Appl. Vol. 6 No. 1 (2019) 115-125.

Lemma 3.3. [6, p. 42] Let $B$ be the set of all the idempotents in $C$, the extended centroid of $R$. Assume $R$ is a $B$-algebra orthogonal complete. For any maximal ideal $P$ of $B, P R$ forms a minimal prime ideal of $R$, which is invariant under any nonzero derivation of $R$.

We will prove the following:

Theorem 3.4. Let $R$ be a semiprime ring, $U$ the left Utumi quotient ring of $R$ and $m, n$ be fixed positive integers. If $R$ admits a nonzero derivation $d$ such that $d(x) \circ_{n} d(y)=x \circ_{m} y$ for all $x, y \in R$, then $R$ is commutative.

Proof. Since $R$ is semiprime and we are given that $d(x) \circ_{n} d(y)=x \circ_{m} y$ for all $x, y \in I$. By Lemma 3.2, $Z(U)=C$, the extended centroid of $R$, and by Lemma 3.1, derivation $d$ can be uniquely extended on $U$, the Utumi quotient ring of $R$. In view of Lee [I2, Theorem 2], $R$ and $U$ satisfy the same differential identities, hence

$$
d(x) \circ_{n} d(y)=x \circ_{m} y
$$

for all $x, y \in U$. Let $B$ be the complete Boolean algebra of idempotents in $C$ and $M$ be any maximal ideal of $B$. Since $U$ is a $B$-algebra which is orthogonal complete (see Chuang [6, p. 42], and Lemma 3.1), by Lemma 3.3, $M U$ is a prime ideal of $U$, which is $d$-invariant. Denote $\bar{U}=U / M U$ and $\bar{d}$ the derivation induced by $d$ on $\bar{U}$, i.e., $\bar{d}(\bar{u})=\overline{d(u)}$ for all $u \in U$. Therefore $\bar{d}$ has in $\bar{U}$ the same property as $d$ on $U$. It is obvious that $\bar{U}$ is prime. Therefore, by Theorem $2.2, \bar{U}$ is commutative. This implies that, for any maximal ideal $M$ of $B,[U, U] \subseteq M U$ and hence $[U, U] \subseteq \bigcap_{M} M U=0$, where $M U$ runs over all prime ideals of $U$. In particular, $[R, R]=0$ and so $R$ is commutative. This completes the proof of the theorem.

Using arguments similar to those used in the proof of the Theorem 3.4 we may conclude with the following (we omit the proof brevity). We can prove

Theorem 3.5. Let $R$ be a semiprime ring, $U$ the left Utumi quotient ring of $R$ and $m, n$ be fixed positive integers. If $R$ admits a nonzero derivation $d$ such that $d(x) \circ_{m} d(y)=(d(x \circ y))^{n}$ for all $x, y \in R$, then $R$ is commutative.

\section{Applications on Banach algebras}

In this section we obtain some results on non-commutative Banach algebras by using the preceding algebraic results.

Here $\mathfrak{A}$ will denote a complex Banach algebra. Let us introduce some well known and elementary definitions for the sake of completeness. By a Banach algebra we shall mean a complex normed algebra $\mathfrak{A}$ whose underlying vector space is a Banach space. The Jacobson 
radical $\operatorname{rad}(\mathfrak{A})$ of $\mathfrak{A}$ is the intersection of all primitive ideals. If the Jacobson radical reduces to the zero element, $\mathfrak{A}$ is called semisimple. In fact any Banach algebra $\mathfrak{A}$ without a unity can be embedded into a unital Banach algebra $\mathfrak{A}_{I}=\mathfrak{A} \oplus \mathbb{C}$ as an ideal of codimension one. In particular we may identify $\mathfrak{A}$ with the ideal $\{(x, 0): x \in \mathfrak{A}\}$ in $\mathfrak{A}_{I}$ via the isometric isomorphism $x \rightarrow(x, 0)$.

In this section we apply the purely algebraic results which are obtained in section 2 and obtain the conditions that every continuous derivation on a Banach algebra maps into the radical. As we have mentioned before, Thomas [18] has generalized the Singer-Wermer theorem by proving that any derivation on a commutative Banach algebra maps the algebra into its radical. This result leads to the question whether the theorem can be proved without any commutativity assumption. There are many papers that the theorem holds without commutativity assumption [14, 16, 17].

Our first result in this section is about continuous derivations on Banach algebras:

Theorem 4.1. Let $\mathfrak{A}$ be a non-commutative Banach algebra with Jacobson radical rad $(\mathfrak{A})$ and $m, n$ be fixed positive integers. Suppose that there exists a nonzero continuous derivation $d: \mathfrak{A} \rightarrow \mathfrak{A}$ such that $d(x) \circ_{n} d(y)-\left(x \circ_{m} y\right) \in \operatorname{rad}(\mathfrak{A})$ for all $x, y \in \mathfrak{A}$, then $d$ maps into the radical of $\mathfrak{A}$.

Proof. We have given that $d(x) \circ_{n} d(y)-\left(x \circ_{m} y\right) \in \operatorname{rad}(\mathfrak{A})$, for all $x, y \in \mathfrak{A}$. Under the assumption that $d$ is nonzero continuous derivation with Jacobson radical $\operatorname{rad}(\mathfrak{A})$. In [16, Theorem 2.2], Sinclair proved that any continuous derivation of a Banach algebra leaves the primitive ideals invariant. Since the Jacobson radical $\operatorname{rad}(\mathfrak{A})$ is the intersection of all primitive ideals, we have $d(\operatorname{rad}(\mathfrak{A})) \subseteq \operatorname{rad}(\mathfrak{A})$, which means that there is no loss of generality in assuming that $\mathfrak{A}$ is semisimple. Since $d$ leaves all primitive ideals invariant, one can introduce for any primitive ideal $P \subseteq \mathfrak{A}$, a nonzero derivation

$$
d_{P}: \mathfrak{A} / P \rightarrow \mathfrak{A} / P
$$

where $\mathfrak{A} / P=\bar{A}$ is a factor Banach algebra

$$
d_{P}(x+P)=d(x)+P \text { for all } x \in \mathfrak{A} .
$$

Note that every derivation on a semisimple Banach algebra is continuous [10, Remark 4.3]. First, in case $\mathfrak{A} / P$ is commutative, combining this result with the Singer-Wermer theorem gives $d_{P}=0$ since $\mathfrak{A} / P$ is semisimple. We intend to show that $d_{P}=0$ in case when $\mathfrak{A} / P$ is non-commutative. Since the assumption of the theorem gives $d(x) \circ_{n} d(y)-\left(x \circ_{m} y\right) \in \operatorname{rad}(\mathfrak{A})$ for all $x, y \in \mathfrak{A} / P$. Thus by Theorem 2.2 , it is immediate that either $\overline{\mathfrak{A}}$ is commutative or 
Alg. Struc. Appl. Vol. 6 No. 1 (2019) 115-125.

$d_{P}=0$ on $\mathfrak{A} / P$. Consequently $d(\mathfrak{A}) \subseteq P$ for any primitive ideal $P$. Since the radical $\operatorname{rad}(\mathfrak{A})$ of $\mathfrak{A}$ is the intersection of all primitive ideals in $\mathfrak{A}$, we get the required conclusion.

Using arguments similar to those used in the proof of Theorem 4.1, we can prove

Theorem 4.2. Let $\mathfrak{A}$ be a non-commutative Banach algebra with Jacobson radical rad( $\mathfrak{A})$ and $m, n$ be fixed positive integers. Suppose that there exists a nonzero continuous derivation $d: \mathfrak{A} \rightarrow \mathfrak{A}$ such that $d(x) \circ_{m} d(y)-(d(x \circ y))^{n} \in \operatorname{rad}(\mathfrak{A})$ for all $x, y \in \mathfrak{A}$, then $d$ maps into the radical of $\mathfrak{A}$.

In order to prove our last result, we will use the following well-known result concerning semisimple Banach algebra contained in [10, Theorem 4.1].

Lemma 4.3. Every nonzero derivation on a semisimple Banach algebra is continuous.

In view of Theorem 4.1 and Lemma 4.3, we may prove the following theorem in the special case when $\mathfrak{A}$ is a semisimple Banach algebra.

Corollary 4.4. Let $\mathfrak{A}$ be a non-commutative semisimple Banach algebra with Jacobson radical $\operatorname{rad}(\mathfrak{A})$ and $m, n$ be fixed positive integers. Suppose that there exists a nonzero continuous derivation $d: \mathfrak{A} \rightarrow \mathfrak{A}$ such that $d(x) \circ_{n} d(y)-\left(x \circ_{m} y\right) \in \operatorname{rad}(\mathfrak{A})$ for all $x, y \in \mathfrak{A}$, then $d(\mathfrak{A})=0$.

Proof. By the hypothesis that $d$ is continuous. In view of the above Lemma 4.3, every nonzero derivation on a semisimple Banach algebra is continuous. Thus every nonzero derivation on a semisimple Banach algebra leaves the primitive ideals of the algebra invariant. Now by using the same argument as used in the proof of the Theorem 4.1, and the fact that $\operatorname{rad}(\mathfrak{A})=0$, since $\mathfrak{A}$ is semisimple, we get the required result.

We immediately get the following corollary from Theorem 4.2.

Corollary 4.5. Let $\mathfrak{A}$ be a non-commutative semisimple Banach algebra with Jacobson radical $\operatorname{rad}(\mathfrak{A})$ and $m, n$ be fixed positive integers. Suppose that there exists a nonzero continuous derivation $d: \mathfrak{A} \rightarrow \mathfrak{A}$ such that $d(x) \circ_{m} d(y)-(d(x \circ y))^{n} \in \operatorname{rad}(\mathfrak{A})$ for all $x, y \in \mathfrak{A}$, then $d(\mathfrak{A})=0$.

\section{AcKnowledgments}

The authors would like to thank the referee for the careful reading of the manuscript and many useful suggestions. 


\section{REFERENCES}

[1] N. Argaç and H. G. Inceboz, Derivation of prime and semiprime rings, J. Korean Math. Soc. Vol. 46 No. 5 (2009), pp. 997-1005.

[2] M. Ashraf and N. Rehman, On commutativity of rings with derivations, Results Math. Vol. 42 No. 1-2 (2002), pp. 3-8.

[3] K. I. Beidar, W. S. Martindale III and A. V. Mikhalev, Rings with Generalized Identities, Pure and Applied Mathematics, Marcel Dekker 196, New York, (1996).

[4] H. E. Bell and M. N. Daif, On commutativity and strong commutativity-preserving maps, Cand. Math. Bull. Vol. 37 (1994), pp. 443-447.

[5] C. L. Chuang, GPIs having coefficients in Utumi quotient rings, Proc. Amer. Math. Soc. Vol. 103 (1988), pp. $723-728$.

[6] C. L. Chuang, Hypercentral derivations, J. Algebra, Vol. 166 (1994), pp. 34-71.

[7] T. S. Erickson, W. S. Martindale III and J. M. Osborn, Prime nonassociative algebras, Pac. J. Math. Vol. 60 (1975), pp. 49-63.

[8] I. N. Herstein, Center-like elements in prime rings, J. Algebra, Vol. 60 (1979), pp. 567-574.

[9] N. Jacobson, Structure of Rings, Colloquium Publications 37, Amer. Math. Soc. VII, Provindence, RI, (1956).

[10] B. E. Johnson, A. M. Sinclair, Continuity of derivations and a problem of Kaplansky, Amer. J. Math. Vol. 90 (1968), pp. 1067-1073.

[11] V. K. Kharchenko, Differential identities of prime rings, Algebra Logic, Vol. 17 (1979), pp. 155-168.

[12] T. K. Lee, Semiprime rings with differential identities, Bull. Inst. Math. Acad. Sin. Vol. 20 (1992), pp. $27-38$.

[13] W. S. Martindale III, Prime rings satisfying a generalized polynomial identity, J. Algebra, Vol. 12 (1969), pp. 576-584.

[14] M. Mathieu and G. J. Murphy, Derivations mapping into the radical, Arch. Math. Vol. 57 (1991), pp. 469-474.

[15] J. H. Mayne, Centralizing mappings of prime rings, Canad. Math. Bull. Vol. 27 No. 1 (1984), pp. $122-126$.

[16] A. M. Sinclair, Continuous derivations on Banach algebras, Proc. Amer. Math. Soc. Vol. 20 (1969), pp. 166-170.

[17] I. M. Singer and J. Wermer, Derivations on commutative normed algebras, Math. Ann. Vol. 129 (1955), pp. 260-264.

[18] M. P. Thomas, The image of a derivation is contained in the radical, Annals of Mathematics, Vol. 128 No. 3 (1988), pp. 435-460.

[19] X. W. Xu, The power values properties of generalized derivations, Doctor Thesis of Jilin University, Changchun, (2006).

\section{Nadeem ur Rehman}

Department of mathematics 
Alg. Struc. Appl. Vol. 6 No. 1 (2019) 115-125.

Aligarh Muslim University

Aligarh, India.

nu.rehman.mm@amu.ac.in

Shuliang Huang

School of Mathematics and Finance

Chuzhou University

Chuzhou, China.

shulianghuang@163.com

Mohd Arif Raza

Department of Mathematics, Faculty of Science and Arts-Rabigh

King Abdulaziz University

Jeddah, Kingdom of Saudi Arabia.

arifraza03@gmail.com 